\title{
Surgical Therapy of Early Carcinoma of the Esophagus
}

\author{
Michael Pauthner Thomas Haist Markus Mann Dietmar Lorenz
}

Department of General and Visceral Surgery, Sana Klinikum Offenbach GmbH, Teaching Hospital of the University Medicine of Frankfurt am Main, Offenbach, Germany

\section{Keywords}

Early esophageal cancer - Prognostic risk factors .

Esophagectomy · Lymphadenectomy

\section{Summary}

Background: The modern therapy of early esophageal carcinomas ( $\mathrm{pT} 1$ ) requires an excellent cooperation between experienced gastroenterologists, pathologists, and esophageal surgeons. While endoscopic resection (ER) is accepted as the standard curative treatment for mucosal esophageal carcinomas, submucosal tumors are regarded as a strict indication for surgery. There is an ongoing discussion about the operative approach and the extent of lymph node dissection in these cases. Methods: A literature review was performed to evaluate the operative treatment of early esophageal cancer. In view of oncological risk factors, treatment strategies, and operative procedures, current studies are summarized and compared to the results of our own center. Results and Conclusion: In early esophageal cancer, lymph node involvement is the only independent risk factor for survival and recurrence rates. There is evidence that infiltrated lymph nodes $(\mathrm{N}+)$ are significantly correlated with tumor infiltration depth, lymphovascular (L1) and microvascular invasion (V1), and poor tumor differentiation (G3). Several studies suggest that early squamous cell carcinomas (eSCCs) and early adenocarcinomas (eACs) have a different tumor biology and therefore need a different treatment strategy. While eSCCs in stage $\mathrm{m} 1$ and $\mathrm{m} 2$ can be cured by ER, tumors infiltrating the submucosal layer (sm1-3) show a high rate of lymph node metastasis (LNM); thus, surgical resection (SR) is clearly indicated. In tumors with invasion into the deep mucosa (m3) the risk of LNM is up to $11 \%$; however, reliable data are rare and the type of therapy should be discussed with the patients individually. In eACs, ER is the standard curative treatment for all mucosal tumors (m1-m4) and sm1 tumors with low-risk constellation (G1, L0, VO, R0).
All high-risk sm1 tumors and those with deeper submucosal infiltration (sm2, sm3) show a high rate of LNM and require SR. The standard operative procedure for early esophageal carcinomas is an Ivor-Lewis esophagectomy with radical, at least two-field lymphadenectomy.

(C) 2015 S. Karger GmbH, Freiburg

\section{Introduction}

In Europe, the incidence of esophageal cancer is about 4.5 cases/100,000/year. While the incidence of squamous cell carcinomas (SCCs) remains stable, adenocarcinomas (ACs) show the highest rise of relative incidence of all cancers in the Western world, and its prognosis is still poor $[1,2]$. Long-term survival is strongly correlated with the cancer stage at the time of diagnosis. Thus, ongoing efforts are being made to improve early endoscopic detection of esophageal cancer.

For a long time radical surgical resection (SR) was the treatment of choice in all patients with esophageal cancer regardless of its stage; however, esophageal surgery still carries a mortality rate of $2-5 \%$ even in high-volume centers while morbidity rates range from 20 to $50 \%$ [3-5].

It has been 15 years since endoscopic resection (ER) for earlystage esophageal carcinoma has been reported in larger series [6]. Meanwhile, this treatment approach has been widely accepted since numerous studies demonstrated its efficacy, its safety, and excellent long-term results for tumors limited to the mucosa [7-10].

Naturally, ER cannot be curative if lymph node metastases (LNM) are present. Therefore, reliable criteria are necessary for patients who can be cured by ER alone and for those who require surgery with adequate lymphadenectomy. There is evidence that these criteria differ between early adenocarcinomas (eAC) and early squamous cell carcinomas (eSCC) so that an individual strategy for both tumor entities is necessary.

\section{KARGER \\ Fax +497614520714

() 2015 S. Karger GmbH, Freiburg

$1662-6664 / 15 / 0315-0326 \$ 39.50 / 0$
Dr. med. Michael Pauthner

Klinik für Allgemein- und Viszeralchirurgie

Sana Klinikum Offenbach GmbH

Starkenburgring 66, 63069 Offenbach, Germany

michael.pauthner@sana.de 


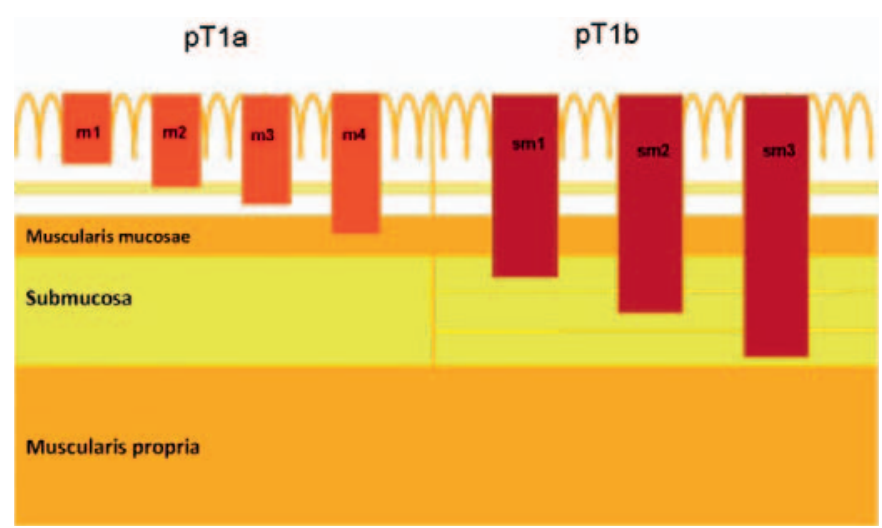

Fig. 1. Cross-section of the esophagus wall with tumor infiltration depth

\section{Histopathological Workup}

Early esophageal carcinomas are defined as tumors limited to the mucosa (pT1a) and submucosa (pT1b), regardless of the presence of LNM. In 2001, the Japanese Society of Esophageal Disease introduced a classification in which the invasion of eSSC was subdivided into six successive layers of the mucosa ( $\mathrm{m} 1, \mathrm{~m} 2$, and $\mathrm{m} 3$ ) and submucosa ( $\mathrm{sm} 1, \mathrm{sm} 2$, and $\mathrm{sm} 3$ ) [11]. This classification found broad acceptance in specialized esophageal centers and proved to have good inter- and intraobserver reproducibility in Western centers as well. In approximately $70-80 \%$ of Barrett's esophagus a second (neo-)muscularis mucosae can be detected so that a subdivision of the mucosa into four levels (m1-m4) was proposed (fig. 1) [12].

Following an ER, invasion depth and histological pattern of the neoplastic lesion are analyzed by an experienced pathologist. Depth of tumor invasion, the resection margins (R), grading $(G)$, and the presence of lymphovascular (L) or microvascular invasion (V) must be evaluated to decide if the endoscopic therapy was curative or if an SR is necessary.

The classification of the operated specimens is performed according to the 7th edition of the UICC/TNM classification of malignant tumors. For the final diagnosis the highest tumor stage of either the ER or the SR specimen must be taken [13].

\section{Staging}

Prior to ER, all patients with early esophageal cancer must have a diagnostic high-resolution videoendoscopy with chromoendoscopy first. In addition, an endoscopic ultrasound (EUS) is performed to determine the invasion depth of the tumor and the regional lymph node status. If suspicious lymph nodes (larger than $10 \mathrm{~mm}$, hypoechoic, or rounded appearance) are detected, EUSguided puncture is carried out. Patients selected for surgery and those with suspicious lymph nodes during EUS require a high-resolution computed tomography scan of the chest including the upper abdomen and an abdominal ultrasound to rule out metastasis or a second neoplasia.

\section{Treatment Strategies and Indication for Surgery}

There is evidence that eSCCs and eACs have different tumor characteristics, especially once the tumor infiltrates into the submucosa [14]. A recent meta-analysis including 2,831 patients with surgery for $\mathrm{pT} 1 \mathrm{~b}$ tumors reported that eSCCs show a higher rate of LNM in sm 1 and sm 2 tumors compared to eACs. In this study, the rate of LNM in sm3 stages was $>50 \%$ in both tumor types. Overall, eSCCs seemed to be more aggressive than eACs and showed substantially higher rates of LNM ( $\mathrm{pN}+45 \mathrm{vs.} 26 \%$ ) as well as lymphovascular or microvascular invasion ( $\mathrm{L}+57$ vs. $37 \%$; $\mathrm{V}+40$ vs. $18 \%$ ) [15]. As a consequence, eSCCs and eACs require different treatment strategies and should not be analyzed together.

eSCC

Several studies from Asian and Western centers showed that ER of eSCC is the treatment of choice if the tumor infiltrates the mucosa up to $\mathrm{m} 2$ stage. In these cases the risk of LNM is less than $2 \%$ which is lower than the mortality after SR even in high-volume centers $[2-5,16,17]$.

Once the tumor infiltrates into the deep mucosal (m3) and submucosal layers (sm1-sm3), the rate of LNM increases considerably (m3: up to $11 \%$, sm1: 27\%, sm2: $37 \%$, sm3: $55 \%$ ) [15, 18].

A study from Shanghai with 189 patients who had radical surgery for an eSCC showed no LNM in $\mathrm{m} 1$ and $\mathrm{m} 2$ tumors but $11 \%$ LNM in the $\mathrm{m} 3,24 \%$ in the sm1, 20.5\% in the sm2, and $43.8 \%$ in the $\mathrm{sm} 3$ subgroup. In this report, lymphovascular infiltration (L1) and invasion of the sm3 layer were independent risk factors for LNM [19].

In a retrospective analysis from Japan with 295 patients treated by ER and SR for eSCCs, a direct correlation between tumor depth, the risk of LNM, and the chance of cure was confirmed. The authors observed no LNM in the stages $\mathrm{m} 1$ and $\mathrm{m} 2$ but found $9 \%$ in $\mathrm{m} 3,16 \%$ in $\mathrm{sm} 1,35 \%$ in $\mathrm{sm} 2$, and $62 \%$ in $\mathrm{sm} 3$ tumors. The risk of distant metastases was $0 \%$ in $\mathrm{m} 1-\mathrm{m} 3$ and $\mathrm{sm} 1$ tumors as well as $9 \%$ in $\mathrm{sm} 2$ and $13 \%$ in $\mathrm{sm} 3$ stages. The tumor-specific 5-year survival rates (5Y-SR) were $100 \%$ for mucosal tumors, $90.9 \%$ for $\mathrm{sm} 1$ tumors, but only $78.8 \%$ for sm 2 tumors and $66.8 \%$ for sm 3 tumors. Vascular and lymphatic infiltrations (V1/L1) were independent risk factors for LNM [20].

In conclusion, eSCCs in the stages $\mathrm{m} 1$ and $\mathrm{m} 2$ with no lymphovascular or microvascular invasion in the ER specimen can be treated endoscopically with curative intent. In $\mathrm{m} 3$ cases, the type of therapy should be individually discussed with the patients considering oncologic benefit, operative risk, and comorbidities.

All patients with high-risk mucosal tumors (L1/V1) and all submucosal tumors without evidence of LNM should be selected for radical SR unless a very proximal tumor close to the upper esophageal sphincter is present or the patients' condition is not good enough for surgery. In these cases, patients are treated by definitive radiochemotherapy.

Patients with eSCCs and lymphonodular infiltration should be treated by means of preoperative chemoradiation. The high risk of LNM in submucosal eSCCs and the reduced tumor-specific 5Y-SR, 
Table 1. Criteria for esophagectomy in pT1 squamous cell carcinoma (Wiesbaden/ Offenbach Center)

\author{
Infiltration depth sm1-sm3 \\ Infiltration depth m3: individual discussion \\ Lymphovascular or microvascular infiltration \\ (L1, V1) \\ Incomplete basal resection (R1) \\ Lymph node metastases $(\mathrm{N}+)$ : preoperative \\ radiochemotherapy
}

Table 2. Criteria for esophagectomy in pT1 adenocarcinoma (Wiesbaden/Offenbach Center)
Infiltration depth sm2, sm3

Infiltration depth sm1 high-risk (G3, L1, V1)

Lymphovascular or microvascular infiltration (L1, V1)

Incomplete basal resection (R1)

Lymph node metastases $(\mathrm{N}+)$ : preoperative (radio-)chemotherapy especially in sm 2 and sm 3 stages, raise the question whether a multimodality treatment might be favorable in all submucosal SCCs (table 1).

\section{eACs}

ER proved to be a safe and effective treatment of mucosal eACs because the risk of LNM is low [7-10]. A systematic review of 70 studies with 1,874 patients operated for high-grade dysplasia and mucosal AC of the esophagus showed LNM in only 26 patients with pT1a tumors $(1.93 \%)$ and none in high-grade dysplasia. The authors concluded that an SR is not indicated in high-grade dysplasia and mucosal ACs of the esophagus because mortality rates after esophagectomy exceed the risk of LNM [21]. A matched control study of two specialized esophageal centers comparing ER and SR for pTla tumors demonstrated excellent long-term survival rates (median follow-up: 4 years) in both groups but morbidity ( 32 vs. $0 \%)$ and mortality rates (2.6 vs. $0 \%$ ) were remarkably higher after SR. There was a risk of recurrent disease and metachronous dysplasia in the ER group (6.6\%); however, in all of these patients a complete remission was possible with repeated ERs [22].

Even though ER has become the treatment of choice in mucosal eACs, any tumor infiltration of the submucosal layer is still regarded as a strict indication for SR because increasing rates of LNM are described in this situation [14, 15, 23].

Our group demonstrated in 168 patients with SR for eAC that the lymph node status is the only independent risk factor for survival and recurrence rates [24]. The 5Y-SR was $87.1 \%$ for $\mathrm{pN} 0$ tumors and $56.0 \%$ for $\mathrm{N}+$ tumors $(\mathrm{p}<0.001)$. Lymph node infiltration $(\mathrm{N}+)$ was significantly correlated with tumor infiltration depth, lymphovascular (L1) and microvascular invasion (V1), and poor tumor differentiation (G3). We did not find a distinct anatomically defined watershed between tumors infiltrating the deep mucosa $(\mathrm{m} 3 / \mathrm{m} 4)$ and the superficial submucosa $(\mathrm{sm} 1)$, as recently proposed in the literature [25]. Combining the results of all endoscopically and surgically treated patients in our center, the percentage of SR was $8.25 \%$ (32/388 patients) in $\mathrm{m} 3 / \mathrm{m} 4$ tumors and $29.8 \%$ (37/124) in sm1 tumors. Therefore, the operatively resected patients represent a selection of high-risk cases according to our treatment protocol on the basis of the histological patterns.

In our study, the $\mathrm{N}+$ rates in submucosal tumors were $8.1 \%$ in $\mathrm{sm} 1,27.3 \%$ in sm2, and $25 \%$ in sm3. These percentages were confirmed in our latest analysis of more than 200 operatively resected eACs (unpublished data). Compared to the results of a recent meta-analysis [15], the rate of LNM in sm3 stage was remarkably low in our series ( 25 vs. $58 \%$ ). An explanation for this might be that the histopathologic workup in our patients follows a strictly defined scientific protocol and is more precise than it usually is in surgical series with bulky operative specimens. In most of our cases, we have twice as much information from the ER and the SR specimens. One could argue that a greater probability of missing the real (more advanced) tumor stages in the surgical specimens simulates lymph node involvement in earlier tumor stages in other, mostly retrospective series.

In contrast to the study by Hölscher et al. [14], we found no significant correlation between survival and the mucosal or submucosal infiltration of the tumor. An explanation might be that there was a relatively high percentage of eSSCs in the mixed cohort of the Cologne study.

In our study, the analysis of the risk factors is based on the lymph node status at the time of operation. The follow-up of our patients demonstrated that the risk of further developing metastases (overall metastatic rate) is significantly correlated with the infiltration depth and the lymphovascular invasion (L1) (unpublished data).

In conclusion, after a diagnostic ER in all eACs an individual decision for surgery based on the histopathology of the specimen has to be made depending on the presence of risk factors (L1, V1). There is a major subgroup of sm1 tumors without those risk factors which can be treated by ER in curative intent $[26,27]$. All tumors with sm2 and sm3 infiltration depth should be surgically resected because the risk of LNM exceeds the risk of the operation by far. Furthermore, all patients after failed ER are referred to surgery if their general health status allows an operation. ER failure in our center was defined as positive basal (not radial) margin, technical problems during ER because of failed lifting, repeated local tumor recurrences after seemingly curative ER, LNM or distant metastases during follow-up, and poor healing after ER.

Patients with proven or highly suspicious lymph nodes should be considered for multimodality treatment (perioperative chemotherapy or preoperative radiochemotherapy) (table 2) [2].

\section{Surgical Procedures}

Since ER is the gold standard for high-grade dysplasia and mucosal carcinomas of the esophagus, the risk of LNM in patients with submucosal tumors selected for surgery has significantly increased. As a consequence, the operative procedure does not only have to remove the carcinoma but also has to focus on an appropriate lymph node dissection (LND). 
The standard operative technique for all stages of an esophageal carcinoma is the Ivor-Lewis esophagectomy (IL) with a systematic two-field lymphadenectomy of mediastinal and abdominal lymph nodes. The gastrointestinal passage is usually reconstructed with a gastric pull-up and a high intrathoracic anastomosis. However, this procedure has a notable morbidity and a hospital mortality of up to $5 \%$ even in high-volume centers [3-5]. As an alternative operative technique for early esophageal carcinomas the transhiatal esophagectomy had earlier been used. The procedure has the advantage of avoiding a thoracotomy, thus potentially reducing postoperative complications; however, only a limited LND of the lower mediastinum is possible $[28,29]$.

There is one randomized trial comparing radical IL with limited transhiatal resection for AC of the mid and distal esophagus [30]. No significant overall survival benefit for either approach was found. Compared to limited transhiatal resection, however, IL with radical LND showed an ongoing trend towards a better 5-year survival. Moreover, patients with a limited number of positive lymph nodes in the resection specimen (1-8 lymph nodes) seemed to benefit from the extended procedure (5-year locoregional disease-free survival: 23 vs. $64 \%, p=0.02$ ). Based on this data, transthoracic resection with complete mediastinal LND is to be favored for all submucosal ACs because of the high risk of lymph node involvement. Transhiatal esophagectomy can be considered in patients with an early mucosal AC and a very low risk of LNM who cannot be treated by ER for technical reasons, especially in cases with reduced pulmonary function.

Hölscher et al. [14] reported about 171 patients with early esophageal carcinoma (121 eACs, 50 eSCCs) treated by SR (IL: 161, transhiatal esophagectomy: 10). The rate of LNM was $0 \%$ for 70 mucosal and $34 \%$ for 101 submucosal carcinomas. The $5 \mathrm{Y}$-SR was $82 \%$ for $\mathrm{pN} 0$ and $45 \%$ for $\mathrm{pN}+$ patients $(\mathrm{p}<0.001)$. There was no significant prognostic difference between AC and SCC (5Y-SR: 74 vs. $71 \%)$. The $5 \mathrm{Y}$-SR for $\mathrm{sm} 1$ and $\mathrm{sm} 2$ were similar to the pT1a group (80\%) and significantly better than for sm3 (46\%) (p = 0.008 ), which was the only independent prognostic factor in multivariate analysis $(\mathrm{p}=0.01)$. The results indicate that the prognosis after SR in submucosal tumors can be as good as in mucosal tumors if a radical LND is carried out.

A study from China with 189 patients treated by means of IL and radical D2 lymphadenectomy for eSCCs showed LNM in $33.1 \%$ of the submucosal tumors, and paratracheal nodes were those most frequently involved. 'Skip' metastases occurred in 20 of 49 patients $(40.8 \%)$. The authors concluded that a radical two-field lymphadenectomy with careful upper mediastinal LND should be the standard for submucosal SCCs [19]. A Japanese group reported on the long-term results of 105 patients with submucosal esophageal cancer (98 SCCs) who underwent transthoracic esophagectomy with extended three-field or two-field lymphadenectomy. LNM were present in 38 patients (36.2\%), of whom 9 patients (23.7\%) had positive cervical nodes. The overall 5- and 10-YSRs were 74.4 and $57.4 \%$, respectively. There were no differences in survival rates between patients with LNM and those without ( $\mathrm{p}=$ 0.9809). Multivariate analysis showed other primary malignancies to be the only independent prognostic factor [31]. These excellent results are a further argument for radical LND in early esophageal carcinomas.

In experienced hands, an IL can be done minimally invasively using a laparoscopic and thoracoscopic approach or a laparoscopic-assisted approach with an open thoracotomy (hybrid operation). The majority of studies show that minimally invasive esophagectomies (MIE) are associated with a significant reduction of pulmonary complications and blood loss as well as a shorter length of stay on the intensive care unit [28, 32-34]. The oncologic quality (number of lymph nodes dissected, tumor stage) after MIE is comparable to open surgery but reliable data regarding longterm survival is still missing [35]. The pending long-term results of the Dutch multicenter randomized controlled trial should be able to answer this question [36].

The Merendino procedure (MER) - originally implemented as an anti-reflux operation in 1955 [37] - was oncologically modified by the Munich group for the treatment of eACs and published in 2000 [38]. This technique combines the resection of the lesser curve with a reduction of the stomach to its half and an LND around the lesser curvature, the celiac trunk, and the lower mediastinum. The gastrointestinal passage is reconstructed with interposition of an isoperistaltic jejunal segment of $12-15 \mathrm{~cm}$ in length.

As with transhiatal esophagectomy, only a limited LND of the lower mediastinum is possible; thus, the oncologic quality of MER is questionable considering the results of the Dutch randomized trial [30].

In our own experience of 30 oncologic MERs, the number of resected lymph nodes was significantly higher in the IL group compared to the MER group. Postoperative morbidity and mortality after IL and MER were surprisingly comparable (unpublished data). Regarding the quality of life, our group demonstrated that MER is not superior to IL [39]. On the contrary, MER patients suffered from a significant impairment of health-related quality of life 1 year after surgery that gradually improved to the level of IL resection after 2 years. Therefore, we abandoned this operative technique in favor of laparoscopic-assisted IL in 2007.

In conclusion, an IL with radical (at least two-field) lymphadenectomy is the standard operative technique for all stages of esophageal cancer. MIE has advantages over the open procedure regarding short-term outcome while the oncological results seem to be comparable.

\section{Disclosure Statement}

The authors declare no conflicts of interest. 


\section{References}

1 Jemal A, Murray T, Ward E, et al: Cancer statistics. CA Cancer J Clin 2005; 1:10-30.

2 Stahl M, Mariette C, Haustermans K, et al: Oesophageal cancer: ESMO Clinical Practice Guidelines for diagnosis, treatment and follow-up. Ann Oncol 2013; 24(suppl 6):vi51-56.

3 Jamieson GG, Mathew G, Ludemann R, et al: Postoperative mortality following oesophagectomy and problems in reporting its rate. Br J Surg 2004;8:943-947.

4 Viklund P, Lindblad M, Lu M, et al: Risk factors for complications after esophageal cancer resection: a prospective population-based study in Sweden. Ann Surg 2006;2:204-211.

5 Atkins BZ, Shah AS, Hutcheson KA, Mangum JH, Pappas TN, Harpole DH Jr, D’Amico TA: Reducing hospital morbidity and mortality following esophagectomy. Ann Thorac Surg 2004;78:1170-1176; discussion 1170-1176.

6 Ell C, May A, Gossner L, et al: Endoscopic mucosal resection of early cancer and high-grade dysplasia in Barrett's esophagus. Gastroenterology 2000;118:670677.

7 Pech O, May A, Manner H, et al: Long-term efficacy and safety of endoscopic resection for patients with mucosal adenocarcinoma of the esophagus. Gastroenterology 2014;146:652-660.e1.

8 Pech O, Behrens A, May A, et al: Long-term results and risk factor analysis for recurrence after curative endoscopic therapy in 349 patients with high-grade intraepithelial neoplasia and mucosal adenocarcinoma in Barrett's oesophagus. Gut 2008;57:1200-1206.

$\checkmark$ Peters FP, Kara MA, Rosmolen WD, et al: Endoscopic treatment of high-grade dysplasia and early stage cancer in Barrett's esophagus. Gastrointest Endosc 2005; 61:506-514.

10 Conio M, Cameron AJ, Chak A, et al: Endoscopic treatment of high-grade dysplasia and early cancer in Barrett's oesophagus. Lancet Oncol 2005;6:311-321.

11 Japanese Society for Esophageal Diseases: Guidelines for Clinical and Pathologic Studies on Carcinoma in the Esophagus, ed 9. Tokyo, Kanehara \& Co, Ltd, 2010.

12 Stolte M, Kirtil T, Oellig F, et al: The pattern of invasion of early carcinomas in Barrett's esophagus is dependent on the depth of infiltration. Pathol Res Pract 2010;206:300-304.

13 Wittekind C, Meyer H-J: TNM-Klassifikation Maligner Tumoren. Weinheim, Wiley-VCH, 2010.

14 Hölscher AH, Bollschweiler E, Schröder W, et al: Prognostic impact of upper, middle, and lower third mucosal or submucosal infiltration in early esophageal cancer. Ann Surg 2011;254:802-807; discussion 807808.

15 Gockel I, Sgourakis G, Lyros O, et al: Risk of lymph node metastasis in submucosal esophageal cancer: a review of surgically resected patients. Expert Rev Gastroenterol Hepatol 2011;254:802-807; discussion 807808.
6 Pech O, May A, Gossner L, Rabenstein T, Manner H, Huijsmans J, Vieth M, Stolte M, Berres M, Ell C: Curative endoscopic therapy in patients with early esophageal squamous cell carcinoma or high-grade intraepithelial neoplasia. Endoscopy 2007;39:30-35.

17 Ciocirlan M, Lapalus MG, Hervieu V, Souquet JC, Napoléon B, Scoazec JY, Lefort C, Saurin JC, Ponchon T: Endoscopic mucosal resection for squamous premalignant and early malignant lesions of the esophagus. Endoscopy 2007;39:24-29.

18 Eguchi T, Nakanishi Y, Shimoda T, Iwasaki M, Igaki H, Tachimori Y, Kato H, Yamaguchi H, Saito D, Umemura S: Histopathological criteria for additional treatment after endoscopic mucosal resection for esophageal cancer: analysis of 464 surgically resected cases. Mod Pathol 2006;19:475-480.

19 Li B, Chen H, Xiang J, et al: Prevalence of lymph node metastases in superficial esophageal squamous cell carcinoma. J Thorac Cardiovasc Surg 2013;146:1198-1203.

20 Akutsu Y, Uesato M, Shuto K, et al: The overall prevalence of metastasis in T1 esophageal squamous cell carcinoma: a retrospective analysis of 295 patients. Ann Surg 2013;257:1032-1038.

21 Kerry B, Dunbar KB, Spechler JS: The risk of lymphnode metastases in patients with high-grade dysplasia or intramucosal carcinoma in Barrett's esophagus: a systematic review. Am J Gastroenterol 2012;107:850862.

22 Pech O, Bollschweiler E, Manner H, et al: Comparison between endoscopic and surgical resection of mucosal esophageal adenocarcinoma in Barrett's esophagus at two high-volume centers. Ann Surg 2011;254:67-72.

23 DeMeester SR: Evaluation and treatment of superficial esophageal cancer. J Gastrointest Surg 2010;14(suppl 1): S94-S100.

24 Lorenz D, Origer J, Pauthner M, et al: Prognostic risk factors of early esophageal adenocarcinomas. Ann Surg 2014;259:469-476.

25 Raja S, Rice WT, Goldblum JR, et al: Esophageal submucosa: the watershed for esophageal cancer. J Thorac Cardiovasc Surg 2011;142:1403-1411.

26 Manner H, Pech O, Heldmann Y, et al: Efficacy, safety, and long-term results of endoscopic treatment for early stage adenocarcinoma of the esophagus with lowrisk sm1 invasion. Clin Gastroenterol Hepatol 2013;11: 630-635; quiz e45.

27 Manner H, Pech O, Heldmann Y, et al: The frequency of lymph node metastasis in early-stage adenocarcinoma of the esophagus with incipient submucosal invasion (pT1b sm1) depending on histological risk patterns. Surg Endosc 2015;29:1888-1896.

28 Hulscher JB, van Sandick JW, de Boer AG, Wijnhoven BP, Tijssen JG, Fockens P, Stalmeier PF, ten Kate FJ, van Dekken H, Obertop H, Tilanus HW, van Lanschot JJ: Extended transthoracic resection compared with limited transhiatal resection for adenocarcinoma of the esophagus. N Engl J Med 2002;347:1662-1669.
9 Ferguson MK, Celauro AD, Prachand V: Prediction of major pulmonary complications after esophagectomy. Ann Thorac Surg 2011;91:1494-1500; discussion 15001501

30 Omloo JM, Lagarde SM, Hulscher JB, Reitsma JB, Fockens P, van Dekken H, Ten Kate FJ, Obertop H, Tilanus HW, van Lanschot JJ: Extended transthoracic resection compared with limited transhiatal resection for adenocarcinoma of the mid/distal esophagus: fiveyear survival of a randomized clinical trial. Ann Surg 2007;246:992-1000; discussion 1000-1001.

31 Tanaka T, Matono S, Nagano T, et al: Esophagectomy with extended lymphadenectomy for submucosal esophageal cancer: long-term outcomes and prognostic factors. Ann Surg Oncol 2012;19:750-756.

32 Luketich JD, Pennathur A, Awais O, et al: Outcomes after minimally invasive esophagectomy: review of over 1000 patients. Ann Surg 2012;256:95-103.

33 Smithers BM, Gotley DC, Martin I, Thomas JM: Com parison of the outcomes between open and minimally invasive esophagectomy. Ann Surg 2007;245:232-240.

34 Nagpal K, Ahmed K, Vats A, Yakoub D, James D, Ashrafian H, Darzi A, Moorthy K, Athanasiou T: Is minimally invasive surgery beneficial in the management of esophageal cancer? A meta-analysis. Surg Endosc 2010;24:1621-1629.

35 Dantoc MM, Cox MR, Eslick GD: Does minimally invasive esophagectomy (MIE) provide for comparable oncologic outcomes to open techniques? A systematic review. J Gastrointest Surg 2012;16:486-494.

36 Biere SS, van Berge Henegouwen MI, Maas KW, et al: Minimally invasive versus open oesophagectomy for patients with oesophageal cancer: a multicentre, openlabel, randomised controlled trial. Lancet 2012;379: 1887-1892.

37 Merendino KA, Dillard DH: The concept of sphincter substitution by an interposed jejunal segment for anatomic and physiologic abnormalities at the esophagogastric junction; with special reference to reflux esophagitis, cardiospasm and esophageal varices. Ann Surg 1955;3:486-506.

38 Stein HJ, Feith M, Mueller J, et al: Limited resection for early adenocarcinoma in Barrett's esophagus. Ann Surg 2000;6:733-742.

39 Zapletal C, Heesen C, Origer J, Pauthner M, Pech O, Ell C, Lorenz D: Quality of life after surgical treatment of early Barrett's cancer: a prospective comparison of the Ivor-Lewis resection versus the modified Merendino resection. World J Surg 2014;38:1444-1452. 